\title{
Isoelectric Focusing to Quantify Rhodopsin Phosphorylation in Mouse Retina
}

Sowmya Bekshe Lokappa ${ }^{1}$, M. Carter Cornwall ${ }^{2}$ and Jeannie Chen ${ }^{1, *}$

\author{
${ }^{1}$ Department of Physiology and Neuroscience, Zilkha Neurogenetic Institute, University of Southern \\ California, Los Angeles, California, USA; '2Department of Physiology and Biophysics, Boston University \\ School of Medicine, Boston, Massachusetts, USA \\ *For correspondence: jeannie@usc.edu
}

[Abstract] Rhodopsin is a G-protein coupled receptor (GPCR) that mediates vision under dim light. Upon light exposure, rhodopsin is phosphorylated at multiple serine and threonine sites at its carboxylterminus by rhodopsin kinase (GRK1). This, in turn, reduces its ability to activate the visual G-protein transducin. Binding of light-activated, phosphorylated rhodopsin by arrestin (ARR1) fully terminates the catalytic activity of rhodopsin. Quantification of the levels of the differentially phosphorylated rhodopsin species provides definitive information about the role of phosphorylated rhodopsin in visual functions. Isoelectric Focusing (IEF) is a technique which is used to separate ampholytic components, such as proteins, based on their isoelectric point (pl). It is a useful technique used to distinguish protein isoforms and post-translational modifications such as phosphorylation, glycosylation, deamination, and acetylation, due to their effects on the protein's pl. Isoelectric Focusing can provide high resolution of differentially phosphorylated forms of a protein. Though other techniques such as kinase activity assays, phospho-specific antibodies, western blot, enzyme-linked immunosorbent assays (ELISA), radiolabeling and mass spectrometry are used to detect and quantify protein phosphorylation, IEF is a simple and cost-effective method to quantify rhodopsin phosphorylation, as it can readily detect individual phosphorylated forms.

Here we provide a detailed protocol for determining phosphorylated rhodopsin species using the Isoelectric Focusing technique.

Keywords: Rhodopsin, Isoelectric Focusing, Post-translational modification, Phosphorylation, GPCR, Photoreceptors

[Background] Rhodopsin is a seven-helix transmembrane protein covalently linked to a chromophore, 11-cis-retinal, which acts as a powerful antagonist, holding rhodopsin in its inactive state. Photon absorption isomerizes the retinal from cis to trans which then triggers conformational changes in the protein moiety into a catalytically active form. Activated rhodopsin facilitates GTP-GDP exchange in the visual G-protein transducin. Transducin-GTP binds and releases the inhibitory constraint of the gamma subunit on phosphodiesterase 6 (PDE6), which then hydrolyzes the diffusible second messenger cGMP, leading to closure of cation cGMP gated channels localized in the plasma membrane. This collective of reactions, called the phototransduction cascade, provides the first steps of vision and is one of the best characterized G-protein signaling pathways (Molday and Moritz, 2015). 
Like other GPCRs, rapid quenching of receptor activity depends on phosphorylation by a receptor kinase and subsequent binding of arrestin. During rhodopsin deactivation, rhodopsin kinase (GRK1) sequentially phosphorylates six serine/threonine residues contained within residues 334-343 near the C-terminus of mouse rhodopsin: $\underline{S} A \underline{T}$ S $\underline{S} K \underline{I} E \underline{T S}$. These multiple phosphorylation sites confer reproducibility of the rod's quantal response to single photon absorption (Mendez et al., 2000; Doan et al., 2006; Azevedo et al., 2015) which is essential for our ability to see under dim light conditions when photons are sparse. In the absence of ARR1, rhodopsin phosphorylation alone decreases the efficiency of transducin activation (Xu et al., 1997). Even single and double phosphorylations can decrease rhodopsin's catalytic activity by half (Mendez et al., 2000). Therefore, a reliable methodology to quantify rhodopsin phosphorylation will provide important information on how this common posttranslational modification affects phototransduction, and thus visual performance.

Traditionally, the most common method used to detect protein phosphorylation involved the use of radiolabeling. Phosphorylation of rhodopsin by GRK1 has been determined using radiolabeled $\left[\mathrm{Y}^{-}{ }^{32} \mathrm{P}\right]$ ATP (Zhang et al., 1997). Greene et al. (1995) have also shown kinetic and structural analysis of the phosphorylation of rhodopsin by protein kinase $\mathrm{C}$. However, the limitations of the radiolabeling method include sample variability and an inability to quantify pre-bound unlabeled phosphate. Reverse phase column chromatography has been used to separate phospho- and non-phospho mouse rhodopsin peptides, and to study the kinetics of phosphorylation/dephosphorylation (Ohguro and Palczewski, 1995). Phospho-specific antibodies are also routinely used to analyze key targets in normal and disease states. Though useful for initial studies, phospho-specific antibodies fail to detect unphosphorylated species; hence stoichiometric measurements cannot be made. Production of quality phospho-specific antibodies is time-consuming, costly, and also requires prior knowledge of specific phosphorylation sites (Arur and Schedl, 2014).

Mass spectrometry has become a highly sensitive nonradioactive method to analyze protein phosphorylation. Electrospray mass spectrometry has been used to identify sequential phosphorylation of rhodopsin at multiple sites in vitro (Ohguro et al., 1993) and in vivo (Kennedy et al., 2001; Lee et al., 2002). However, major challenges in analyzing protein phosphorylation using mass spectrometry include access to the instrumentation and the possible high cost associated with using the instruments through core facilities. Additionally, mass accuracy of post-translational modifications depends on the resolution of the mass spectrometer used. Some of the intrinsic limitations are: a) mass spectrometry is sensitive in a particular $\mathrm{m} / \mathrm{z}$ (mass-to-charge ratio) range; b) protease cleavage efficiencies vary for different proteins and different domains of same protein; and c) cleaved peptides have different ionization and detection efficiencies (Kim et al., 2016).

Isoelectric Focusing (IEF) is a high-resolution technique used for separating charged molecules, usually proteins or peptides, on the basis of their isoelectric point ( $\mathrm{pl}$ ) within a continuous $\mathrm{pH}$ gradient. $\mathrm{pl}$ is defined as the $\mathrm{pH}$ at which the molecule has no charge. Separation is achieved by initially establishing a stable $\mathrm{pH}$ gradient along the length of the gel using ampholytes. The sample components then travel through the $\mathrm{pH}$ gradient under an electric field until their individual charges reach zero. Proteins move towards the electrode with an opposite charge. The gradient is set up so that negatively 
charged molecules migrate towards decreasing $\mathrm{pH}$, whereas positively charged molecules move towards increasing $\mathrm{pH}$. For this technique, a low concentration polyacrylamide or agarose gel is used as it is only a stabilizing medium and does not participate in the sample separation. IEF can readily detect individual phosphorylated forms, as every additional phosphate increases the negative charge on rhodopsin protein, and hence causes a shift in pl (Berry et al., 2016). Here we describe a simple and cost-effective method to determine phosphorylated rhodopsin species using Isoelectric Focusing (IEF) in a horizontal gel format followed by immunoblot detection.

\section{Materials and Reagents}

1. $2.0 \mathrm{ml}$ sterile round bottom microfuge tubes (USA Scientific, catalog number: 1620-2700)

2. $0.5 \mathrm{ml}$ sterile microfuge tubes (USA Scientific, catalog number: 1605-0000)

3. $10 \mu \mathrm{l}$ Sterile pipette tips (USA Scientific, catalog number: 1111-3810)

4. $200 \mu \mathrm{l}$ Sterile pipette tips (USA Scientific, catalog number: 1111-1810)

5. $1000 \mu \mathrm{l}$ Sterile pipette tips (USA Scientific, catalog number: 1111-2821)

6. $25 \mathrm{ml}$ Serological pipettes (USA Scientific, catalog number: 1072-5410)

7. $15 \mathrm{ml}$ sterile conical centrifuge tubes (VWR International, catalog number: 10025-686)

8. $50 \mathrm{ml}$ sterile conical centrifuge tubes (VWR International, catalog number: 10025-698)

9. Pipettes (Research-includes 3-pack [2-20 $\mu \mathrm{l}, 20-200 \mu \mathrm{l}, 100-1,000 \mu \mathrm{l}]$, Eppendorf, catalog number: EPPR3OP2)

10. Nitrocellulose membrane (Sigma-Aldrich, catalog number: GE10600002)

11. Blotting paper (Thermo Fisher Scientific, catalog number: 88600)

12. Aluminum foil (VWR International, catalog number: 89107-724)

13. $3 \mathrm{~cm}$ plastic Petri dish (Thermo Fisher Scientific, catalog number: 150318)

14. Saran wrap (VWR International, catalog number: 89136-664)

15. Ames' medium (Sigma-Aldrich, catalog number: A1420-10X1L)

16. Acrylamide ReadySol IEF 40\% (GE Healthcare, catalog number: 17-1310-01)

17. 4-(2-hydroxyethyl)-1-piperazineethanesulfonic acid (HEPES) (Omnipur, catalog number: 5320)

18. Sodium fluoride (NaF) (Fischer Scientific, catalog number: S299-100)

19. Adenosine (Sigma-Aldrich, catalog number: A-4036)

20. Phenylmethylsulfonyl fluoride (PMSF) (Sigma-Aldrich, catalog number: P-7626)

21. Ethylenediaminetetraacetic acid (EDTA) (VWR, catalog number: BDH4616)

22. Roche protease inhibitors (Sigma-Aldrich, catalog number: 11873580001)

23. Milli-Q water

24. Liquid nitrogen

25. $95 \% \mathrm{O}_{2} / 5 \% \mathrm{CO}_{2}$ gas cylinder

26. Magnesium chloride ( $\mathrm{MgCl}_{2}$ ) (EMD, catalog number: MX0045-1)

27. Bovine Serum Albumin (BSA)-Fatty acid free (Sigma-Aldrich, catalog number: A-8806)

28. Sodium chloride ( $\mathrm{NaCl})$ (EMD, catalog number: SX0420-3) 
29. Tris- $\mathrm{HCl}$ (Sigma-Aldrich, catalog number: T3253)

30. Tween-20 (Sigma-Aldrich, catalog number: P1379)

31. Nonfat-dried milk (Sigma-Aldrich, catalog number: M7409)

32. n-dodecyl- $\beta$-D-maltoside (DDM) (Avanti Polar Lipids, catalog number: 850520)

33. 11-cis-retinal (National Eye Institute) or 9-cis-retinal (Sigma-Aldrich, catalog number: R5754)

34. Glycerol (Sigma-Aldrich, catalog number: G5516-1L)

35. Glutamic acid (Sigma-Aldrich, catalog number: G-1251)

36. Sodium hydroxide (EMD, catalog number: SX0593-3)

37. Dithiothreitol (DTT) (Research Products International Corp [RPI], catalog number: D11000-1000)

38. Mineral oil (Bio-Rad, catalog number: 1632129)

39. Ammonium persulfate (Sigma-Aldrich, catalog number: A3678)

40. TEMED (Sigma-Aldrich, catalog number: T9281)

41. Rhodopsin antibody RET-P1 (Santa Cruz Biotechnology, catalog number: sc-57433)

42. Peroxidase labeled anti-mouse IgG (Vector Laboratories, catalog number: PI-2000)

43. Pharmalyte 2.5-5 for IEF (GE Healthcare, catalog number: 17-0451-01)

44. Pharmalyte 5-8 for IEF (GE Healthcare, catalog number: 17-0453-01)

45. Sodium phosphate dibasic $\left(\mathrm{Na}_{2} \mathrm{HPO}_{4}\right)$ (Sigma-Aldrich, catalog number: $\left.\mathrm{S} 7907\right)$

46. Potassium phosphate monobasic $\left(\mathrm{KH}_{2} \mathrm{PO}_{4}\right)$ (Sigma-Aldrich, catalog number: P5655)

47. Potassium chloride (KCl) (Sigma-Aldrich, catalog number: P9541)

48. Absolute ethanol (Sigma-Aldrich, catalog number: E7023)

49. Sodium bicarbonate $\left(\mathrm{NaHCO}_{3}\right)$ (Sigma-Aldrich, catalog number: $\left.\mathrm{S} 5761\right)$

50. ECL western blotting detection reagents (GE Healthcare, catalog number: RPN2209)

51. Buffer $A$ (Homogenization buffer) (see Recipes)

52. Buffer B (Regeneration buffer) (see Recipes)

53. Buffer C (Solubilization buffer) (see Recipes)

54. 11-cis-retinal stock (see Recipes)

55. IEF Gel solution (see Recipes)

56. $1 x$ Phosphate buffered saline (PBS) (see Recipes)

57. 1x Tris-buffered saline with Tween-20 (TBST) (see Recipes)

\section{Equipment}

1. Photostimulator: A light bench for photostimulation of the retina

This device (Figure 1) consists of a tungsten-halogen light source, neutral density filters, interference filters, and other optics necessary to focus $500 \mathrm{~nm}$ light of uniform intensity on a spot roughly $5 \mathrm{~mm}$ in diameter, where a Petri dish containing the retina is placed. The intensity, duration and wavelength of light is chosen so as to bleach (photoactivate) a specified fraction of rhodopsin. 
The components that comprise the optical system used for bleaching were all purchased from Thorlabs. Thorlabs components are modular and can be used in a variety of combinations to align all of the optical components. Intense white light is provided by a $6 \mathrm{~V} 100 \mathrm{~W}$ tungstenhalogen lamp of the sort normally connected to laboratory light microscopes. The light source used in the Figure 1 (in-house setup) was taken from a discarded Zeiss microscope, but an illuminator from any other microscope manufacturer will serve the purpose as well. The components of the bleaching apparatus are mounted in the light path on an aluminum plate (Thorlabs; MB1218), which has an array of $1 / 4$ "-20 threaded holes for affixing the components. The components are lined up in a linear fashion to allow adjustment of wavelength and intensity of the bleaching beam. First in line is a filter wheel (Thorlabs; CFW6) with positions for 5 oneinch diameter neutral density filters. The following neutral density filters are used:

NE05B-A $25.0 \mathrm{~mm}$ diameter absorptive ND filter ND $=0.5$

NE10B-A $25.0 \mathrm{~mm}$ diameter absorptive ND filter ND $=1.0$

NE20B-A $25.0 \mathrm{~mm}$ diameter absorptive ND filter ND $=2.0$

NE30B-A $25.0 \mathrm{~mm}$ diameter absorptive ND filter ND $=3.0$

NE40B-A $25.0 \mathrm{~mm}$ diameter absorptive ND filter ND $=4.0$

These filters are mounted in a 1-inch mounting hole in each of the 5 spaces in the wheel. Next in line are two cage plates (Thorlabs; CP02) each with a 1-inch diameter hole to accept optic components. The first component is a 1-inch diameter $500 \mathrm{~nm}$ (green) interference filter (Thorlabs; FD1G). This filter can be changed for another which has different band pass characteristics, depending on the sensitivity of the photoreceptors under study. Such filters are available from Thorlabs. The second component is a 1-inch diameter plano convex lens of focal length $5 \mathrm{~cm}$ (Thorlabs; LA1951-A). These two components are connected together by intermediate optical tubes (Thorlabs; SM1L10). The second of these two cage plates is connected to a cage cube-mounted non-polarizing beam splitter (Thorlabs; CCM1-BS013) that contains a mirror to direct light vertically to a $4 \mathrm{~mm}$ aperture, over which the retina in physiological solution is placed. A variety of miscellaneous mechanical rods and brackets for mounting components to the base plate can also be purchased from Thorlabs. These include a mounting base (Thorlabs; BA2, BA1S) which connects to the base plate, mounting rods (Thorlabs; ER2, ER3) and mounting posts (Thorlabs; TR2, TR1) that connect components together. Normally, a thin opaque plastic plate or cardboard card is placed between the colored filter and the lens to act as a shutter. Because relatively long times of light exposure are normally used, removal and subsequent insertion of the card provides light stimulation of sufficient accuracy.

The photostimulator can be constructed using the following parts (Figure 1):
a. Tungsten-halogen lamp (Thorlabs, catalog number: QTH10B)
b. Aluminum plate (Thorlabs, catalog number: MB1218)
c. Filter wheel with positions for neutral density filters (Thorlabs, catalog number: CFW6) 
d. Neutral density filters (Thorlabs, catalog numbers: NE05B-A; NE10B-A; NE20B-A; NE30BA; NE40B-A)

e. Cage plates (Thorlabs, catalog number: CP02)

f. Interference filter (Thorlabs, catalog number: FD1G)

g. Plano convex lens (Thorlabs, catalog number: LA1951-A)

h. Intermediate optical tube (Thorlabs, catalog number: SM1L10)

i. Cage cube-mounted non-polarizing beam splitter (Thorlabs, catalog number: CCM1-BS013)

j. Mounting base (Thorlabs, catalog numbers: BA2; BA1S)

k. Mounting rods (Thorlabs, catalog numbers: ER2; ER3)

I. Mounting posts (Thorlabs, catalog number: TR1)

2. A black, light-tight Delrin plastic box (Figure 2) Used for incubation of the bleached retinal tissue. Incubation is optionally performed for various periods in darkness following light exposure to test the persistence of rhodopsin phosphorylation in darkness.

3. Fluorescent light $(B \& H$, Porta-trace/Gagne LED light box (11"x18"), catalog number: POLB1118L2)

4. Fluorescent light-Lithonia lighting (Aubuchon Hardware, catalog number: 676883)

5. Homogenizer (Kinematica, Polytron, model: PT1200C)

Note: Three tips are available for Polytron PT-1200C, each optimized for different volumes. We recommend the tip optimized to homogenize $\sim 0.1-5 \mathrm{ml}$ of sample (Kinematica, model: PT-DA 1205/2EC). The tip speed (max.) used is $3.5 \mathrm{~m} / \mathrm{s}$.

6. Hoefer dual gel caster (Hoefer, catalog number: SE6015)

7. Clamp assembly (Hoefer, catalog number: SE6003U)

8. Cams (Hoefer, catalog number: SE6005L)

9. Spacer (Hoefer, catalog number: SE6119-2-.75)

Note: Refer to the Hoefer catalog for protein electrophoresis http://www.harvardapparatus.com/media/harvard/pdf/Hoefer Catalog Protein Electrophoresis .pdf.

10. IEF system (GE Healthcare, Amersham Pharmacia, model: Multiphor II system)

11. Cooling system (GE Healthcare, Amersham Pharmacia, model: MultiTemp III system)

12. Electrofocusing strips (GE Healthcare, Amersham Pharmacia, catalog number: 18100440)

13. Sample application strip (GE Healthcare, catalog number: 18-1002-26)

14. Power supply (Bio-Rad, model: PowerPac 3000)

15. Centrifuge

16. $4{ }^{\circ} \mathrm{C}$ refrigerator

17. $-80^{\circ} \mathrm{C}$ freezer

18. Dim red light bulb (Amazon, Delta 1 Brightlab Universal Red Junior Safelight $11 \mathrm{~W}$ bulb, catalog number: 35110)

19. Nutator (VWR International, catalog number: 82007-202) 
20. Curved tweezers (Electron Microscopy Sciences, catalog number: 78752-11)

21. Scalpel (Electron Microscopy Sciences, catalog number: 72042-11)

\section{$\underline{\text { Software }}$}

1. ImageJ (NIH/https://imagej.nih.gov/ii/)

\section{Procedure}

\section{A. Sample preparation}

Note: All materials and equipment that come in contact with samples must be clean. Minimize handling and manipulation of samples.

Rhodopsin is a chromoprotein made up of opsin and 11-cis-retinal. When the eye is exposed to light, the 11-cis-retinal component of rhodopsin is converted to all-trans-retinal, resulting in a fundamental change in the configuration of the rhodopsin. The change in configuration causes opsin to dissociate from retinal, resulting in bleaching during which rhodopsin is deactivated through receptor phosphorylation. Light-exposed retinae contain a mixed population of phosphorylated and unphosphorylated rhodopsin. Complete dark adaptation can only occur when all rhodopsin has been dephosphorylated, a process that requires $>3 \mathrm{~h}$ in complete darkness. Variable time periods are used to capture differentially phosphorylated species. Dark adapted retinae not exposed to light is the control as all rhodopsin species are unphosphorylated.

1. Perform all the procedures involved in dissection, preparation, and Isoelectric Focusing under infrared light or dim red light. We use Delta 1 Brightlab Universal Red Junior Safelight 11 W bulb in our dark room as the source of dim red light. Rhodopsin phosphorylation can be examined by performing experiments both in situ in intact mice or in retinae that had been isolated and then incubated for various times in darkness. Processing of dark-adapted tissue serves as a control for bleached samples. All the mice are dark adapted overnight before exposure to light.

2. For each experimental condition, sacrifice mice under dim red light or infrared light by cervical dislocation. Mice can be of any strain or gender and at 3-12 weeks of age at the time of experiment. Wild-type strain, like C57BL/6J, is included for comparison.

3. To examine rhodopsin phosphorylation in isolated retinae, first dissect the retina from the eye (Video 1). After euthanizing, place the mouse on a flat clean surface and protrude the eye out by applying pressure around the socket. Place the curved tweezers underneath the eyeball and gently pinch the eyeball using your dominant hand. Hold scalpel in your nondominant hand and gently cut the cornea from top to bottom (dorsal to ventral). Put pressure under the bottom of the eye using curved tweezers and gently pinch so that the lens pops out. Remove the lens gently using tweezers and continue to put pressure from the bottom to pull out the retina. Place the retina in buffered Ames' medium (1.9 $\mathrm{g} / \mathrm{L} \mathrm{NaHCO} 3$ bubbled with $95 \% \mathrm{O}_{2} / 5 \% \mathrm{CO}_{2}$ ) (Ames and Nesbett, 1981). Phosphorylation determination is made under five different experimental 
conditions: a) Freeze the dark adapted retinae and store it at $-80^{\circ} \mathrm{C}$ (Dark), b) Dark adapt the retinae, bleach and immediately freeze and store at $\left.-80^{\circ} \mathrm{C}(0 \mathrm{~h}), \mathrm{c}\right)$ Dark adapt the retinae, bleach, incubate in darkness for $1 \mathrm{~h}$, immediately freeze and store at $\left.-80^{\circ} \mathrm{C}(1 \mathrm{~h}), \mathrm{d}\right)$ Dark adapt the retinae, bleach, incubate in darkness for $2 \mathrm{~h}$, immediately freeze and store at $-80{ }^{\circ} \mathrm{C}(2 \mathrm{~h})$, e) Dark adapt retinae, bleach, incubate in darkness for $3 \mathrm{~h}$, immediately freeze and store at $-80^{\circ} \mathrm{C}(3 \mathrm{~h})$. Further process these samples for IEF analysis. For bleaching, place the retinae in buffered Ames' medium ( $1.9 \mathrm{~g} / \mathrm{L} \mathrm{NaHCO} 3$ bubbled with $95 \% \mathrm{O}_{2} / 5 \% \mathrm{CO}_{2}$ ) in the center of a 5 $\mathrm{mm}$ diameter "O" ring affixed to the center of a $3 \mathrm{~cm}$ plastic Petri dish. This dish is centered on the stage of a calibrated photostimulator, as illustrated in Figure 1.

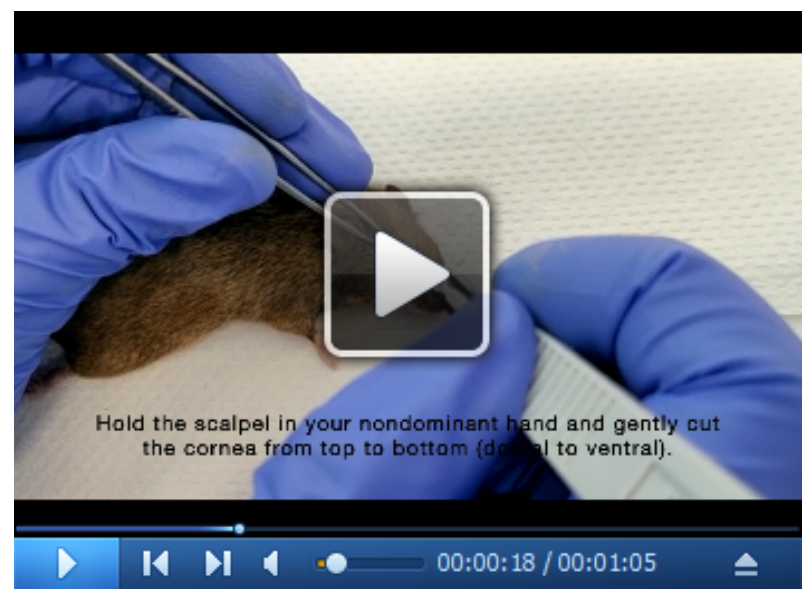

Video 1. Retinal Dissection. This video was made at University of Southern California according to guidelines from the University of Southern California on Animal Care, and approved by the Animal Research Ethics Board of University of Southern California under IACUC protocol ID 11853 and 20575.

The photostimulator consists of a variable intensity tungsten-halogen light source, a $500 \mathrm{~nm}$ (green) interference filter (to set the color of bleaching light to that at which rhodopsin is most sensitive), and neutral density filters (to adjust the intensity of the light). Thus, this apparatus defines the intensity, wavelength, and time of light stimulation to provide a precise bleaching of photopigment. The light spot is sufficiently large and of uniform intensity to provide uniform exposure to the entire retina. 


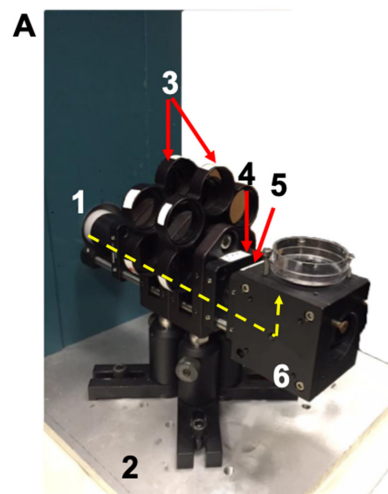

Figure 1. Photographs of the photostimulator. A. Parts of the photostimulator; 1-Tungstenhalogen light source. Light path is highlighted in yellow; 2-Aluminum plate on which components are mounted; 3-Two filter wheels in which neutral density filters are mounted; 4-Cage plate with $500 \mathrm{~nm}$ (green) interference filter mounted next to the neutral density filters; 5-Cage plate with plano convex lens; 6-Cage cube-mounted non-polarizing beam splitter located under the tissue chamber. B. A front surface mirror oriented at $45^{\circ}$ to the beam axis, which reflects light upward to fill a $5 \mathrm{~mm}$ aperture over which the Petri dish containing the retina is centered.

4. The fraction of photopigment bleached by the photostimulator is calculated according to the relation:

$$
F=1-\exp (-I P t)
$$

where,

$F$ is the fraction of pigment bleached;

$I$ is the intensity of the bleaching light;

$P$ is the photosensitivity of the visual pigment $\left(5.7 \times 10^{-9} \mu \mathrm{m}^{2}\right.$ per molecule) measured in situ at the wavelength of peak absorbance;

$t$ is the time of light exposure (Woodruff et al., 2004).

Time of light exposure and light intensity should be adjusted to bleach at a rate of $0.03 \%$ of the total pigment per second.

5. After bleaching, incubate the retinae in darkness in a light-tight Delrin chamber (Figure 2) in Ames' medium to which $1.9 \mathrm{~g} / \mathrm{L} \mathrm{NaHCO}_{3}$ and $3.33 \mathrm{~g} / \mathrm{L}$ fatty-acid-free bovine serum albumin have been added. Solution in this plastic chamber is equilibrated with a $95 \% \mathrm{O}_{2} / 5 \% \mathrm{CO}_{2}$ gas mixture, which sets the $\mathrm{pH}$ at 7.4. In cases where indicated, $10 \mu \mathrm{M}$ 11-cis-retinal is added to facilitate rhodopsin regeneration (11-cis-retinal stock preparation, see Recipes).

6. Incubate retinae in darkness for variable periods up to $3 \mathrm{~h}$ at room temperature (RT). At the end of the incubation period, remove retinae in darkness and place it in individual $2 \mathrm{ml}$ microfuge tubes. 


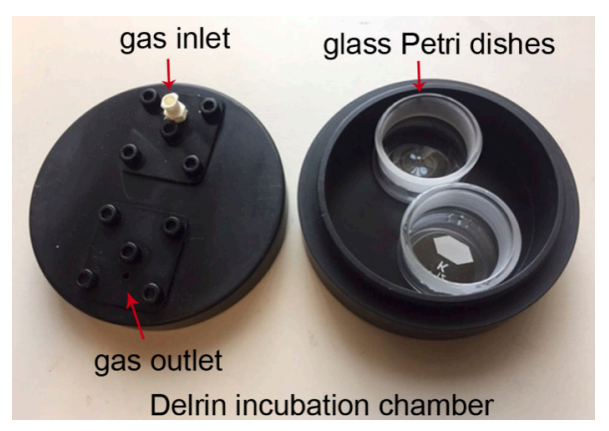

Figure 2. Custom-made Delrin light-tight chamber. Lid at left shows marked gas inlet (top) and outlet (bottom). The lower portion of the chamber is shown to the right. Here, two glass incubation dishes are shown. These dishes are filled with Ames' medium in which the retinal tissue is immersed during incubation in darkness.

7. Microfuge tubes are rapidly frozen in liquid nitrogen, wrapped in light-tight aluminum foil, labeled, and stored at $-80^{\circ} \mathrm{C}$.

For in vivo experiments dark adapt the mice overnight. Expose the mice to fluorescent light for 15 min, which will result in $~ 90 \%$ bleach (Berry et al., 2016). The in-house setup for bleaching experiment is shown in Figure 3. Photostimulator is not required for bleaching in in vivo experiments. Phosphorylation determination is made under five different experimental conditions. Dissect the retina from dark adapted mice and freeze the retina immediately (Dark). Also expose 4 different mice to white light for $15 \mathrm{~min}$ and place the mice in darkness for the indicated times $(0 \mathrm{~h}, 1 \mathrm{~h}, 2 \mathrm{~h}$, $3 \mathrm{~h}$ ), then dissect and freeze the retina and store at $-80^{\circ} \mathrm{C}$. Further process these retinal samples for IEF analysis.

A

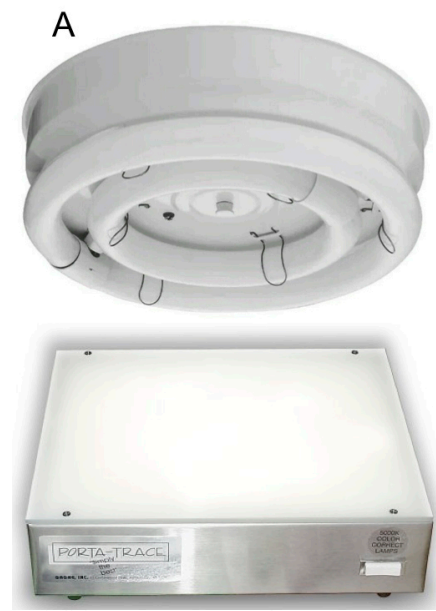

B

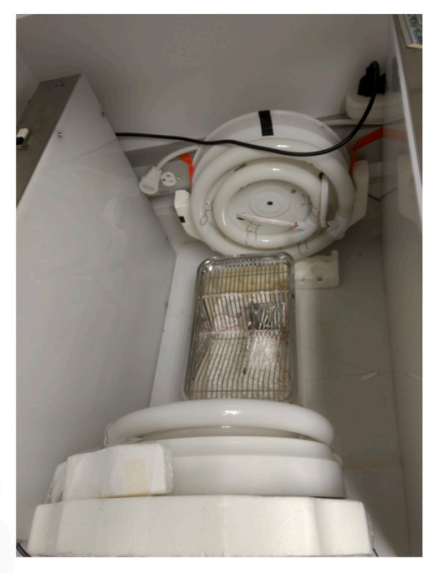

Figure 3. In-house setup for bleaching for in vivo experiment. A. Fluorescent lights. B. Fluorescent light source setup in lab. Mice are placed in a box in the center and exposed to light. 


\section{Process samples for IEF analysis}

1. Thaw the frozen retinae and homogenize retinae in $400 \mu$ of Buffer $A$ (homogenization buffer, see Recipes) using a Polytron ( $30 \mathrm{~s})$.

2. Centrifuge the sample for $15 \mathrm{~min}\left(19,000 \times \mathrm{g}, 4^{\circ} \mathrm{C}\right)$, and discard the supernatant.

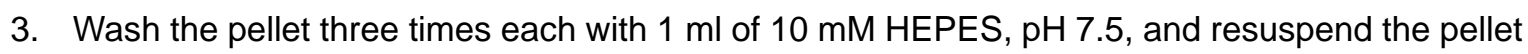
in $1 \mathrm{ml}$ of Buffer B (regeneration buffer, see Recipes).

4. Add excess 9-cis or 11-cis-retinal to the sample ( $900-1,200 \mathrm{pmol})$ (11-cis-retinal or 9-cis-retinal stock preparation, see Recipes).

5. Incubate the sample overnight at $4{ }^{\circ} \mathrm{C}$ on a nutator.

6. Next morning, centrifuge the sample for $15 \min \left(19,000 \times g, 4^{\circ} \mathrm{C}\right)$, and discard the supernatant.

7. Wash the pellet three times each with $1 \mathrm{ml}$ of $10 \mathrm{mM}$ HEPES, $\mathrm{pH} 7.5$, and re-suspend the pellet in $100 \mu \mathrm{l}$ of cold Buffer $\mathrm{C}$ (solubilization buffer, see Recipes) for $3 \mathrm{~h}$ at $4{ }^{\circ} \mathrm{C}$.

8. Centrifuge the sample and transfer supernatant to a $0.5 \mathrm{ml}$ microfuge tube.

9. Add glycerol to the supernatant to $50 \%$ final concentration. To prepare $10 \mu \mathrm{l}$ of final sample for loading on the gel, mix $5 \mu \mathrm{l}$ of supernatant sample with $5 \mu \mathrm{l}$ of glycerol.

10. Load the final sample onto the prepared acrylamide gel as described in Procedure B.

B. IEF gel preparation and electrophoresis (Figure 4)

1. Assemble the Hoefer gel casting apparatus according to the manufacturer's instructions (Figures 4A-4B).

2. Connect the IEF electrophoresis system to the cooling system as per manufacturer's instructions and set the temperature to $10^{\circ} \mathrm{C}$.

3. Prepare $20 \mathrm{ml}$ of IEF gel solution (see Recipes).

4. Swirl the solution gently to mix the components and de-gas the solution not more than 2 min to avoid premature polymerization.

5. Transfer the gel solution to the casting apparatus using $25 \mathrm{ml}$ serological pipette (Figure 4C).

6. Allow at least $90 \mathrm{~min}$ for the gel to polymerize.

7. Gently lift one of the glass plates using the plate separation tool so that gel sticks to the other glass plate.

Note: If gel sticks to the glass plate, clean the glass plate and coat one of the glass plates with repel silane.

8. Saturate electrolyte strips with $0.04 \mathrm{M}$ glutamic acid at the anode and $1 \mathrm{M}$ sodium hydroxide at the cathode, and place directly on the exposed surface of the IEF gel. Make sure that the electrolyte strips are correctly placed and fully in contact with the gel. The length of the electrolyte strips should not exceed the length of the gel.

Note: Gel swells around electrode strips, especially at the cathode. It is a normal phenomenon. Blot the strips with blotting paper occasionally if it interferes with the run.

9. Apply mineral oil over the Amersham Pharmacia Multiphor II IEF system cooling platform and place the glass plate on it with the gel exposed face upward (Figure 4D). 
Note: Mineral oil is applied over the cooling platform of the IEF system so that there is good contact between the glass plate and the cooling plate to allow even cooling.

10. Gently place the electrodes of the platinum wire on the electrolyte strips and install the safety lid.

11. Pre-focus the gel for $40 \mathrm{~min}$ at $23 \mathrm{~W}$ constant power (to initiate $\mathrm{pH}$ gradient) using Bio-Rad PowerPac 3000 high voltage power supply.

12. After pre-focusing for $40 \mathrm{~min}$, remove the safety lid and place the silicone sample application strip on the gel near the anode electrolyte strip. Maintain a distance of $1 \mathrm{~cm}$ between sample application strip and the anode electrolyte strip to protect proteins from exposure to extreme $\mathrm{pH}$ (Figure 4D).

13. Replace the electrolyte strips with fresh solution (if the strip appears dry) and load protein sample onto the gel using silicone sample application strip.

14. Close the electrophoresis cell and carry out Isoelectric Focusing for $2 \mathrm{~h}$ at constant $23 \mathrm{~W}$ or till the voltage does not exceed $3,000 \mathrm{~V}$.

A
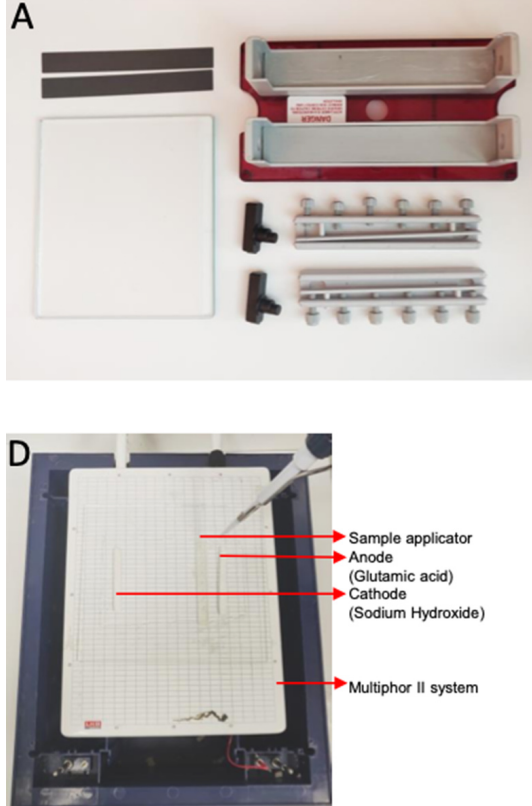

B
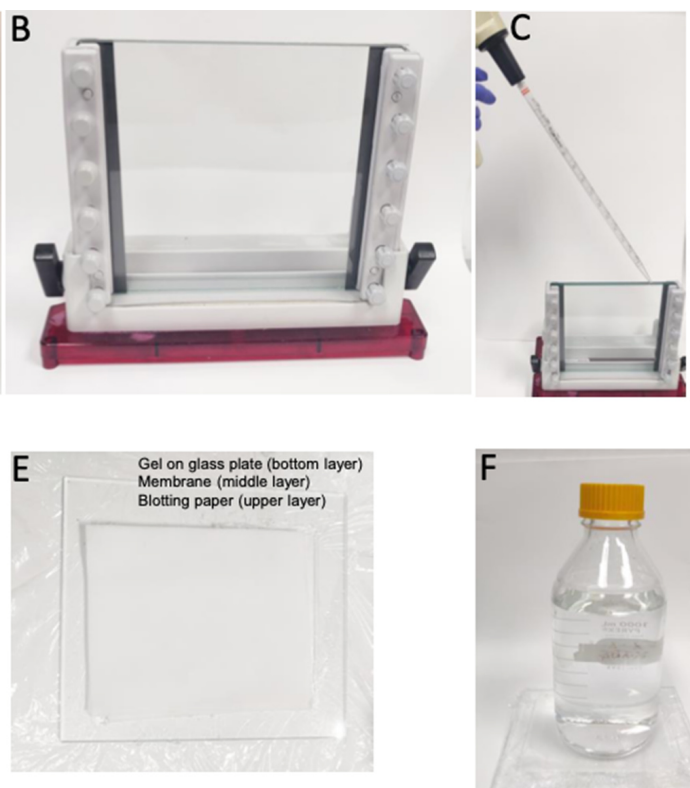

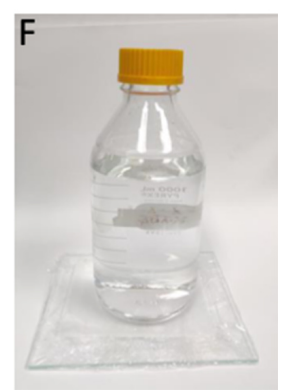

Figure 4. IEF gel casting and electrophoresis. A. Hoefer gel casting apparatus. B. Assembled gel casting unit. C. Pouring gel solution using serological pipette. D. Gel plate on Multiphor IEF system. Anodic strip is soaked in $0.04 \mathrm{M}$ glutamic acid and placed on the gel. Similarly, cathodic strip is soaked in $1 \mathrm{M}$ sodium hydroxide and placed on the gel. Sample applicator is also placed on the gel and gently sample is loaded using micropipette. E. Orientation of gel, membrane, and blotting paper for protein transfer from gel to membrane. F. Protein blotting by capillary method.

C. Preparing gel for immunoblot analysis

1. Remove the gel from the electrophoresis system and remove the electrolyte strips from the gel. 
2. Rinse the gel with $1 x$ phosphate buffered saline (PBS) (see Recipes).

3. Cut the nitrocellulose membrane to the size of the gel, wet the membrane with $1 x$ PBS, and place it on the gel. Rinse blotting paper in 1x PBS and place it on the membrane (Figure 4E).

4. Cover the whole glass plate together with its contents with saran wrap, and invert the glass plate, and place a weight on the glass plate. Transfer proteins onto nitrocellulose membrane by capillary method for 45 min (Figure 4F).

5. Place the membrane in the blocking solution (5\% Nonfat-dried milk in 1x TBST) (see Recipes) for $1 \mathrm{~h}$ at RT. Wash the membrane with $1 \mathrm{x}$ TBST for $10 \mathrm{~min}$. Repeat twice with fresh buffer. Place the membrane in the primary antibody solution (Rhodopsin antibody, RET-P1 in 5\% BSA, $1 \times$ TBST) overnight at $4{ }^{\circ} \mathrm{C}$. Next day, wash the membrane with TBST for 10 min. Repeat twice with fresh buffer. Place the blot in the secondary antibody solution (Peroxidase labeled antimouse IgG, in 1\% BSA, 1x TBST). Again, wash the membrane with TBST for 10 min. Repeat twice with fresh buffer. Visualize the band using ECL system. Quantify the intensity of the bands using NIH ImageJ (Berry et al., 2016). Figure 5 shows immunoblot quantification of rhodopsin phosphorylation.

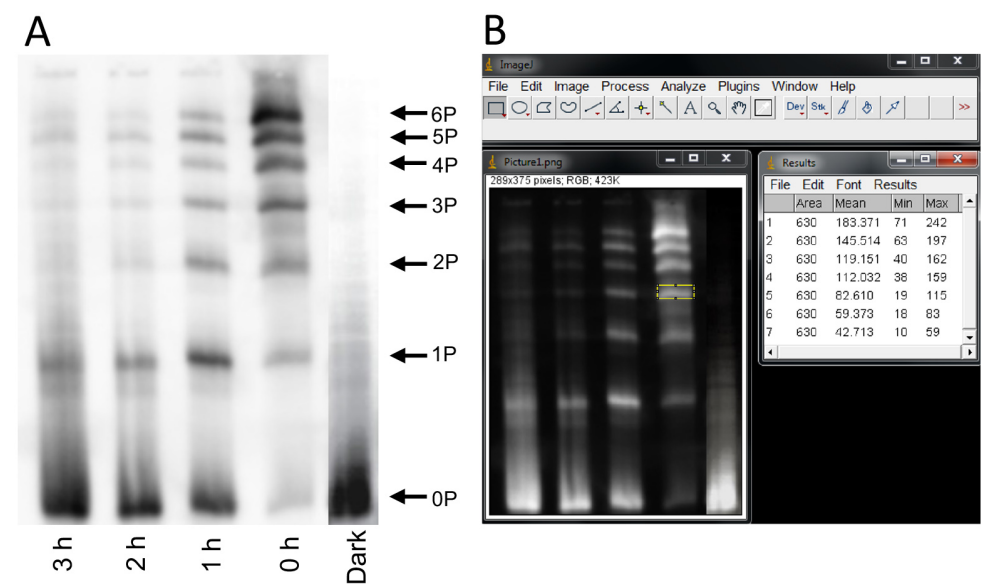

Figure 5. Immunoblot quantification of rhodopsin phosphorylation using ImageJ. A. Immunoblot of rhodopsin carried out after Isoelectric Focusing of rhodopsin from solubilized mouse retinae. Rhodopsin is multiply phosphorylated in vitro in intact rods and in vivo, and the amount of rhodopsin phosphorylation is indicated by the multiple bands representing differently phosphorylated species of rhodopsin. OP corresponds to unphosphorylated rhodopsin. The number next to ' $\mathrm{P}$ ' (1P, 2P, 3P, 4P, 5P and 6P) corresponds to the number of covalently added phosphate groups to rhodopsin. B. Quantification of the intensity of the band using ImageJ. Screen shot of the inverted image (TIFF format) is shown. The bands are selected using the rectangle icon and the values are measured under the "Analyze" command.

\section{Data analysis}

To quantify the signal from the phosphorylated species on the immunoblot, the image of the gel is 
saved as a grayscale TIFF file. The image is inverted using any imaging software so that the background is black and the bands from the gel are white. The inverted image is imported into ImageJ (Fiji). Alternatively, the original grayscale image can be inverted using ImageJ under the "Edit" command. The bands are selected using the rectangle, and the pixel values are measured under the "Analyze" command (command+M). The mean intensity value is recorded for each band. Signals from all bands within a sample are summed to provide the value for total rhodopsin for that sample, and the fractions for each phosphorylated species are calculated by dividing the signal for that species over the total signal.

\section{Recipes}

1. Buffer $A$ (Homogenization buffer)

25 mM HEPES, pH 7.5

$100 \mathrm{mM}$ EDTA

$50 \mathrm{mM} \mathrm{NaF}$

5 mM Adenosine

$1 \mathrm{mM}$ PMSF

$1 x$ Protease inhibitor

Milli-Q water

2. Buffer $B$ (Regeneration buffer)

10 mM HEPES, pH 7.5

\section{$0.1 \mathrm{mM}$ EDTA}

$50 \mathrm{mM} \mathrm{NaF}$

5 mM Adenosine

$1 \mathrm{mM}$ PMSF

$1 \mathrm{mM} \mathrm{MgCl} 2$

2\% BSA

1x Protease inhibitor

Milli-Q Water

3. Buffer $\mathrm{C}$ (Solubilization buffer)

20 mM HEPES, pH 7.5

$0.1 \mathrm{mM}$ EDTA

$50 \mathrm{mM} \mathrm{NaF}$

5 mM Adenosine

$1 \mathrm{mM}$ PMSF

$1 \mathrm{mM} \mathrm{MgCl} 2$

$10 \mathrm{mM} \mathrm{NaCl}$

$1 \% \mathrm{n}$-dodecyl- $\beta$-D-maltoside (DDM)

$1 \mathrm{mM}$ DTT 
1x Protease Inhibitor

Milli-Q water

4. 11-cis-retinal stock

a. Both the stock and powder are wrapped in light-tight aluminum foil and stored at $-80^{\circ} \mathrm{C}$

b. The dissolved retinal stock is stable at $-80{ }^{\circ} \mathrm{C}$ for 3 months whereas the retinal in powder form is more stable.

c. To make a working stock solution, shake a small amount of 11-cis-retinal powder $(<1 \mathrm{mg})$ in a microfuge tube under dim red light conditions and dissolve it in absolute ethanol (100$200 \mu \mathrm{l})$.

d. Perform UV spectrometry with appropriate dilution (typically 1:500-1:1,000) using absolute ethanol as a blank.

11-cis-retinal concentration $(c)$ is calculated as:

$$
c=\frac{O D_{380} I}{\varepsilon_{380}}
$$

where,

$I=1 \mathrm{~cm}$ path length

$\varepsilon_{380}=24,900 \mathrm{M}^{-1} \mathrm{~cm}^{-1}$

Prepare a concentrated stock solution of $\sim 5$ to $10 \mathrm{mM}$ of 11-cis-retinal to avoid exposing the protein to greater than $1 \%$ absolute ethanol during reconstitution procedure.

9-cis-retinal stock

The procedure is similar to the preparation of 11-cis-retinal stock. 9-cis-retinal concentration (c) is calculated as:

$$
c=\frac{O D_{373} I}{\varepsilon_{373}}
$$

where,

$I=1 \mathrm{~cm}$ path length

$\varepsilon_{373}=36,100 \mathrm{M}^{-1} \mathrm{~cm}^{-1}$

5. IEF Gel solution (volume $=20 \mathrm{ml}$ )

This recipe is sufficient for casting one gel of $160 \times 140 \times 1 \mathrm{~mm}$

$40 \%$ Acrylamide solution

$2.5 \mathrm{ml}$

$1 \%$ n-dodecyl- $\beta$-D-maltoside (DDM)

$0.2 \mathrm{~g}$

$13.33 \%$ glycerol

$2.67 \mathrm{ml}$

Pharmalyte $\mathrm{pH}$ 2.5-5

$0.76 \mathrm{ml}$ 

Pharmalyte $\mathrm{pH}$ 5-8
$0.508 \mathrm{ml}$
Ammonium persulfate $(22.8 \mathrm{mg} / \mathrm{ml})$
$0.2 \mathrm{ml}$
TEMED
$0.02 \mathrm{ml}$

Make up the volume to $20 \mathrm{ml}$ with water

6. $1 \times$ Phosphate buffered saline (PBS)

$137 \mathrm{mM} \mathrm{NaCl}$

$10 \mathrm{mM} \mathrm{Na}_{2} \mathrm{HPO}_{4}$

$1.8 \mathrm{mM} \mathrm{KH}_{2} \mathrm{PO}_{4}$

$2.7 \mathrm{mM} \mathrm{KCl}$

Adjust the $\mathrm{pH}$ to 7.4

7. 1x Tris-buffered saline with Tween-20 (TBST)

$50 \mathrm{mM}$ Tris- $\mathrm{HCl} \mathrm{pH} 7.6$

$150 \mathrm{mM} \mathrm{NaCl}$

$0.1 \%$ Tween 20

Note: Recipes 1, 2, 3 and 5 should always be made fresh. Stocks of HEPES, EDTA, and BSA can be prepared and stored at $4{ }^{\circ} \mathrm{C}$. Stocks of NaF, PMSF and DTT can be prepared and stored at $20{ }^{\circ} \mathrm{C}$. Stocks of $\mathrm{NaCl}, \mathrm{MgCl}_{2} 1 \times$ PBS and $1 \times$ TBST can be prepared and stored at RT.

\section{Acknowledgments}

This work was supported by NIH R01 EY12155 to J.C. and NIH EY01157 to M.C.C. This protocol was adapted from Adamus et al., 1993. We are grateful to Ms. Natalie Chen, Ms. Kasey Rose and Dr. Sravani Ramisetty (USC, Los Angeles, USA) for their help with recording the retinal dissection video. We also thank Ms. Yun Yao (USC, Los Angeles, USA) for her help with troubleshooting IEF methodology in the lab and Ms. Natalie Chen for proofreading the manuscript.

\section{Competing interests}

The authors declare no competing financial interests.

\section{Ethics}

The use of mice in these experiments was in accordance with the National Institutes of Health guidelines and the Institutional Animal Care and Use Committee (IACUC) of our respective universities. The IACUC protocol approval ID for the animal experiments are 11853 (Validity- May 2020) and 20575 (Validity-June 2020). 


\section{References}

1. Adamus, G., Arendt, A., Hargrave, P. A., Heyduk, T. and Palczewski, K. (1993). The kinetics of multiphosphorylation of rhodopsin. Arch Biochem Biophys 304(2): 443-447.

2. Ames, A., 3rd and Nesbett, F. B. (1981). In vitro retina as an experimental model of the central nervous system. J Neurochem 37(4): 867-877.

3. Arur, S. and Schedl, T. (2014). Generation and purification of highly specific antibodies for detecting post-translationally modified proteins in vivo. Nat Protoc 9(2): 375-395.

4. Azevedo, A. W., Doan, T., Moaven, H., Sokal, I., Baameur, F., Vishnivetskiy, S. A., Homan, K. T., Tesmer, J. J., Gurevich, V. V., Chen, J. and Rieke, F. (2015). C-terminal threonines and serines play distinct roles in the desensitization of rhodopsin, a $\mathrm{G}$ protein-coupled receptor. Elife 4: e05981.

5. Berry, J., Frederiksen, R., Yao, Y., Nymark, S., Chen, J. and Cornwall, C. (2016). Effect of rhodopsin phosphorylation on dark adaptation in mouse rods. J Neurosci 36(26): 6973-6987.

6. Doan, T., Mendez, A., Detwiler, P. B., Chen, J. and Rieke, F. (2006). Multiple phosphorylation sites confer reproducibility of the rod's single-photon responses. Science 313(5786): 530-533.

7. Greene, N. M., Williams, D. S. and Newton, A. C. (1995). Kinetics and localization of the phosphorylation of rhodopsin by protein kinase C. J Biol Chem 270(12): 6710-6717.

8. Kennedy, M. J., Lee, K. A., Niemi, G. A., Craven, K. B., Garwin, G. G., Saari, J. C. and Hurley, J. B. (2001). Multiple phosphorylation of rhodopsin and the in vivo chemistry underlying rod photoreceptor dark adaptation. Neuron 31(1): 87-101.

9. Kim, M. S., Zhong, J. and Pandey, A. (2016). Common errors in mass spectrometry-based analysis of post-translational modifications. Proteomics 16(5): 700-714.

10. Lee, K. A., Craven, K. B., Niemi, G. A. and Hurley, J. B. (2002). Mass spectrometric analysis of the kinetics of in vivo rhodopsin phosphorylation. Protein Sci 11(4): 862-874.

11. Mendez, A., Burns, M. E., Roca, A., Lem, J., Wu, L. W., Simon, M. I., Baylor, D. A. and Chen, J. (2000). Rapid and reproducible deactivation of rhodopsin requires multiple phosphorylation sites. Neuron 28(1): 153-164.

12. Molday, R. S. and Moritz, O. L. (2015). Photoreceptors at a glance. J Cell Sci 128(22): 40394045.

13. Ohguro, H. and Palczewski, K. (1995). Separation of phospho- and non-phosphopeptides using reverse phase column chromatography. FEBS Lett 368(3): 452-454.

14. Ohguro, H., Palczewski, K., Ericsson, L. H., Walsh, K. A. and Johnson, R. S. (1993). Sequential phosphorylation of rhodopsin at multiple sites. Biochemistry 32(21): 5718-5724.

15. Woodruff, M. L., Lem, J. and Fain, G. L. (2004). Early receptor current of wild-type and transducin knockout mice: photosensitivity and light-induced $\mathrm{Ca}^{2+}$ release. $J$ Physiol 557(Pt 3): 821-828.

16. Xu, J., Dodd, R. L., Makino, C. L., Simon, M. I., Baylor, D. A. and Chen, J. (1997). Prolonged photoresponses in transgenic mouse rods lacking arrestin. Nature 389(6650): 505-509. 
17. Zhang, L., Sports, C. D., Osawa, S. and Weiss, E. R. (1997). Rhodopsin phosphorylation sites and their role in arrestin binding. J Biol Chem 272(23): 14762-14768. 